\title{
Frequencies of Lipopolysaccharide Core Types among Clinical Isolates of Escherichia coli Defined with Monoclonal Antibodies
}

\author{
A. P. Gibb, G. R. Barclay, I. R. Poxton, and F. di Padova
}

Department of Medical Microbiology, University Medical School, and Edinburgh and South-East Scotland Blood Transfusion Centre, Royal Infirnary, Edinburgh. United Kingdom, and Preclinical Research,

Sandoz Pharma, Basel, Swizerland

\begin{abstract}
Mouse monoclonal antibodies (MAbs) specific for the lipopolysaccharide (LPS) core types R1, R2, and R3 of Escherichia coli and a cross-reactive MAb that binds to the LPS core of almost all $E$. coli were used in ELISA to determine the frequency of cores resembling R1, R2, and R3 in strains of $E$. coli isolated from clinical samples (blood and urine specimens) and from the feces of asymptomatic individuals. Of the 180 wild-type isolates, 123 were assigned to $R 1$ core type, 14 to $\mathrm{R} 2$, and 18 to $\mathrm{R} 3$. Twenty-five wild-type $E$. coli isolates could not be assigned to a particular core type and may have either an R4 or K12 core or a previously unrecognized core type. R1 core type was associated with $O$ types $1,4,6,8$, and 18 and with $K 1$ or $K 5$ capsules. $R 3$ was associated with 015. 075 isolates could be of either R1 or R2 core type.
\end{abstract}

The lipopolysaccharide (LPS) core region of Escherichia coli has a conserved overall structure, with an inner 2-keto-3deoxy-octonate-heptose region and an outer hexose region. Most of the variation that does occur is in the outer part of the core, furthest from lipid A. In wild-type smooth E. coli. the core region can occur in at least four different forms, known as $\mathrm{R} 1, \mathrm{R} 2$, $\mathrm{R} 3$, and $\mathrm{R} 4$ [1-3]. The naturally rough strain $K 12$ has a different core region [4].

The $\mathrm{O}$ antigen of smooth LPS may mask some of the epitopes and phage attachment sites in the core region. The chemical, serologic, and phage typing methods that have been used to define the different core types of $E$. coli are therefore not directly applicable to wild-type smooth strains. The frequency with which these different core types occur in wild-type $E$. coli is therefore not known.

Little is known about the association between core types and $O$ serotypes of $E$. coli, except that the original $\mathrm{R} I$ and $\mathrm{R} 2$ rough mutants were both derived from $\mathrm{O} 8$ strains [1] and that the original R3 mutant was derived from an OIII strain [2]. There is no published evidence on the relationship of LPS core types to capsular types or to virulence. Information about the distribution of $\mathrm{O}$ and $\mathrm{K}$ antigens has proved to be useful, for example in identifying and understanding enteropathogenic strains [5] and in suggesting novel therapeutic approaches to bacteremia [6]. Information about core types might be equally interesting.

We therefore set out to produce a panel of mouse monoclo-

\footnotetext{
Received 11 February 1992: revised 21 May 1992.

Financial support: Sandoz Pharma.

Reprints or correspondence: Dr. A. P. Gibb, Department of Medical Microbiology, University Medical School, Teviot Place, Edinburgh, EH8 9AG, UK.

The Journal of Infectious Diseases 1992;166:1051-7 (c) 1992 by The University of Chicago. All rights reserved. 0022-1899/92/6605-0014\$01.00
}

nal antibodies (MAbs) that would react with specific LPS core types of $E$. coli and other gram-negative bacilli with related core structures. We wished to use these MAbs as a means of determining the LPS core types of a collection of wild-type E. coli.

\section{Materials and Methods}

Bacteria and bacteriophages. E. coli R I (F470), R2 (F576), R3 (F653), R4 (2513) and K12 (2131) and rough-specific phages FO, Br10, C21, 6SR, and T4 were obtained from G. Schmidt (Forschunginstitut, Borstel, Germany). E. coli O1, O2, O4, O6, O12, O15, O18, and O75 were obtained from A. S. Cross (Walter Reed Army Institute, Washington, DC). Phages specific for $E$. coli capsular types K1 ( $\phi$ K IGS) and K5 $(\phi \mathrm{K} 5 \mathrm{DG})$ were obtained from A. P. Roberts (Charing Cross and Westminster Medical School, London). Blood culture and urine isolates were obtained from the Clinical Bacteriology Laboratory, Department of Medical Microbiology, Edinburgh University, from routine clinical specimens. The blood culture isolates were all of the gram-negative organisms isolated in a I-year period. Twenty-one fecal isolates of E. coli were obtained, each from a separate healthy volunteer. Rough mutants of Salmonella minnesota (R60), Salmonella typhimurium (1542), Klebsiella pneumoniae (M10B), and Pseudomonas aeruginosa (PAC608) and wild-type smooth Shigella sonnei (colicin types $4 / 2$ and $2 / 1$ ) and Shigella flexneri (serotypes la and 3) were freeze-dried stock cultures held in our laboratory.

MAbs. MAbs were produced by standard methods [7]. The details of immunization schedules and selection procedures are to be published elsewhere. All were reactive in ELISA with polymyxin-complexed rough LPS [8]. All MAb preparations were supernatants of hybridoma cell cultures grown in RPMI 1640 supplemented with 5\% fetal calf serum. Antibody concentration was in the range of $10-50 \mu \mathrm{g} / \mathrm{mL}$.

ELISA. Overnight nutrient broth cultures of bacteria were washed and resuspended in saline to give an OD of 0.5 at 525 $\mathrm{nm}$ and heated for $30 \mathrm{~min}$ at $100^{\circ} \mathrm{C}$. Cells were then diluted 
$1 / 20$ in coating buffer (sodium carbonate-bicarbonate, $\mathrm{pH} 9.6$, with $0.05 \%$ sodium azide) and $100 \mu \mathrm{L} /$ well was added to Nunc (Roskilde, Denmark) Polysorb strips. After overnight incubation at room temperature, plates were washed three times with wash buffer $(0.05 \%$ Tween 20 and $0.05 \%$ sodium azide in PBS, $\mathrm{pH} 7.4$ ) and postcoated with $5 \%$ (wt/vol) bovine serum albumin (BSA) in coating buffer overnight at room temperature. Plates were then washed and stored at $-20^{\circ} \mathrm{C}$.

MAbs were diluted $1 / 250$ in dilution buffer $(4 \%[\mathrm{wt} / \mathrm{vol}] \mathrm{poly}$ ethylene glycol, $0.05 \%$ sodium azide, and $0.05 \%$ tween 20 in PBS, pH 7.4). Diluted MAb (100 $\mu \mathrm{L})$ was added to wells and incubated for $90 \mathrm{~min}$ at $37^{\circ} \mathrm{C}$. Plates were washed three times, and urease-conjugated anti-mouse immunoglobulin (Zymed, Cambridge, UK; diluted $1 / 500$ in dilution buffer with $0.5 \%$ BSA, $100 \mu \mathrm{L} /$ well) was added and incubated for a further 90 min. Plates were then washed three times in wash buffer and three times in distilled water; urease substrate solution (Seralab, Crawley Down, UK; $100 \mu \mathrm{L} /$ well) was added and plates were incubated for $90 \mathrm{~min}$ at room temperature and read at $590 \mathrm{~nm}$. An $\mathrm{OD}$ of $>0.8$ was considered positive.

$P A G E$ and immunobloting. LPS was prepared by proteinase $\mathrm{K}$ (protease type XI; Sigma, Poole, UK) digestion of lysed bacteria [9] and separated on $14 \%$ polyacrylamide gels [10] with SDS omitted from stacking and separating buffers. Gels were then silver stained [9] or blotted to nitrocellulose membrane (0.2- $\mu \mathrm{m}$ pore size) [11]. Blots were blocked with $3 \%(\mathrm{wt} / \mathrm{vol})$ gelatin in TRIS-buffered saline, $\mathrm{pH} 7.5$ (TBS), incubated for $3 \mathrm{~h}$ in a $1 / 10$ dilution of MAb in 1\% gelatin in TBS followed by a 1-h incubation with horseradish peroxidase anti-mouse IgG conjugate (ICN, High Wycombe, UK; diluted 1/1000), and developed with horseradish peroxidase color reagent (Bio-Rad, Hemel Hempstead, UK).

Serum sensitivity of bacteria. Serum from one donor was stored at $-70^{\circ} \mathrm{C}$. This serum had IgG antibodies to LPS core at a level equal to the 65 th centile of the normal population [8]. Overnight broth cultures were washed and resuspended in complement fixation test (CFT) buffer (Oxoid, Basingstoke, UK) at room temperature to an OD of 0.5 at $525 \mathrm{~nm}$. This suspension was then diluted $1 / 5000$ in CFT buffer, and $10 \mu \mathrm{L}$ was added to $70 \mu \mathrm{L}$ of CFT buffer and $20 \mu \mathrm{L}$ of freshly thawed serum or heated $\left(56^{\circ} \mathrm{C}, 30 \mathrm{~min}\right)$ serum. After $1 \mathrm{~h}$ at $37^{\circ} \mathrm{C}$, duplicate 20 $\mu \mathrm{L}$ samples were spread on the surface of nutrient agar plates, and colonies were counted after overnight incubation. If the number of colony-forming units in the fresh serum was $<10 \%$ of the number in the heated serum, the organism was considered to be serum sensitive.

O typing. E. coli isolates from blood and urine cultures were screened for common $\mathrm{O}$ antigens, by ELISA with absorbed $\mathrm{O}$ typing sera (Difco, Detroit) to O1, O2, 04, O6, O7, O12, O15, $\mathrm{O} 18, \mathrm{O} 22$, and $\mathrm{O} 75$ (diluted 1/1000) and by tube agglutination at a final dilution of $1 / 80$ with unabsorbed sera to 08 and 09 (Statens Seruminstitut, Copenhagen). O types were confirmed by conventional tube agglutination [12].

Phage typing. Nutrient agar plates were surface inoculated by flooding with overnight broth culture and allowed to dry. Phage suspension $(10 \mu \mathrm{L})$ was dropped onto the agar and incubated overnight. A clear zone of lysis was scored as sensitive.
Table 1. Reactivity of selected monoclonal antibodies (MAbs) in ELISA.

\begin{tabular}{lccccccccc}
\hline & \multicolumn{8}{c}{ Reactivity with E. coli strain } \\
\cline { 2 - 8 } MAb & R1 & R2 & R3 & R4 & K12 & O12 & O15 & O18 \\
\hline SZ 43 3.4.8 & + & - & - & - & - & - & - & + \\
H4 361.23 & - & + & - & - & - & + & - & - \\
W4 434.07 & - & - & + & - & - & - & + & - \\
SZ 27 150.3 & + & + & + & + & + & + & + & + \\
\hline
\end{tabular}

NOTE. + , strong reactivity $(\mathrm{OD}>0.8)$; - , no or weak reactivity $(\mathrm{OD}$ $<0.15$ ).

Statistics. All $P$ values are two-tailed results calculated by Fisher's exact test.

\section{Results}

Selection of core-specific MAbs. A preselected panel of LPS-reactive MAbs was tested by ELISA against heat-killed $E$. coli rough strains $\mathrm{R} 1, \mathrm{R} 2, \mathrm{R} 3, \mathrm{R} 4$, and $\mathrm{K} 12 ; E$. coli smooth strains $\mathrm{O} 1, \mathrm{O} 2, \mathrm{O} 4, \mathrm{O} 6, \mathrm{O} 12, \mathrm{O} 15, \mathrm{O} 18$, and $\mathrm{O} 75$; and the rough mutants of $K$. pneumoniae, $P$. aeruginosa, $S$. minnesota, and $S$. typhimurium. MAbs that reacted with a restricted range of $\mathbf{R}$ types and also with some smooth strains of $E$. coli were thought likely to be useful in core typing wild-type smooth strains. Four such distinct patterns of reactivity were observed with more than one antibody. Rl-specific MAbs (e.g., SZ 43 3.4.8) reacted with $\mathrm{R} 1$ and with $E$. coli O1, O2, O4, O6, O18, and O75; R2-specific MAbs (e.g., H4 361.23) reacted with $\mathrm{R} 2$ and $\mathrm{O} 12$; R3-specific MAbs (e.g., W4 434.07) reacted with $\mathrm{R} 3$ and $\mathrm{O} 15$; cross-reactive MAb SZ 27150.3 reacted with all the $E$. coli rough and smooth strains and with the Salmonella rough mutants but not with the Klebsiella or Pseudomonas rough mutants (table 1).

Other patterns were observed, including a group of MAbs that reacted with $\mathrm{R} 2$ and $\mathrm{K} 12$, with $\mathrm{R} 2$ and the Salmonella rough mutants, or with all of these strains. However, no MAb reacted with $\mathrm{K} 12$ or $\mathrm{R} 4$ but not with other rough mutants. None of the MAbs used in this study reacted with the Klebsiella or Pseudomonas rough mutants tested.

The specificity of MAbs for LPS core was confirmed, for the MAbs given as examples above, by immunoblotting against LPS (proteinase K extracts) from $E$. coli R1, R2, R3, $\mathrm{R} 4, \mathrm{~K} 12, \mathrm{O} 12, \mathrm{O} 15$, and $\mathrm{O} 18$. The $\mathrm{O} 12$, O15, and $\mathrm{O} 18$ strains were chosen as they seemed, from the ELISA data, to represent smooth strains with $\mathrm{R} 2, \mathrm{R} 3$, and $\mathrm{R} 1$ core types, respectively. As seen in figure 1, the immunoblots showed reactivity of each MAb with a band corresponding to the LPS core of each of the organisms with which it reacted in ELISA. 

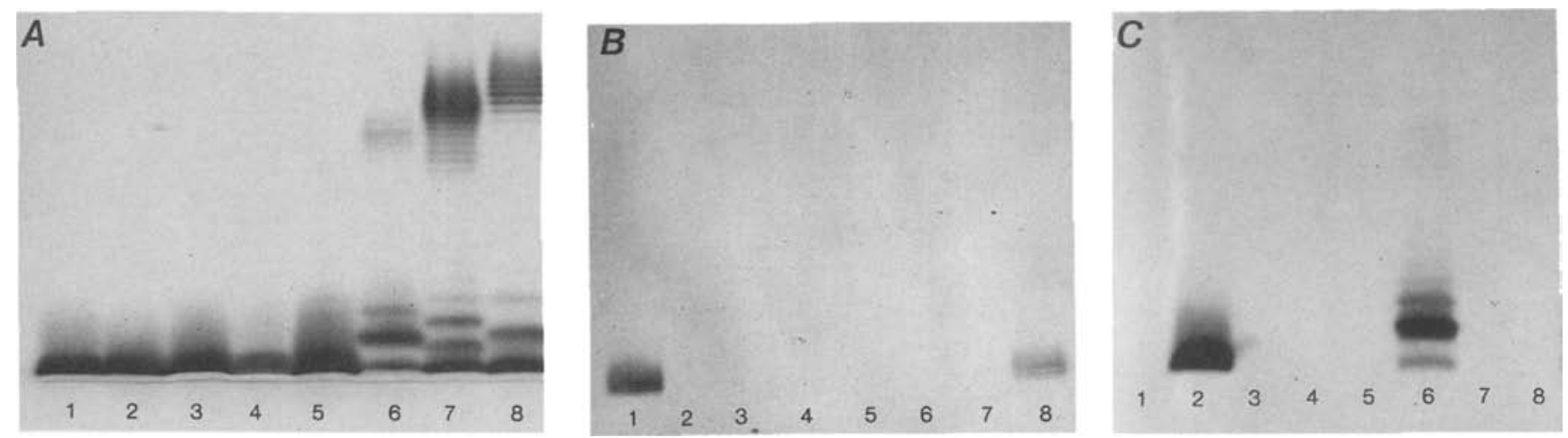

Figure 1. Proteinase $\mathrm{K}$ preparations of LPS from E. coli. Lanes 1-8: Strains R1, R2, R3, R4, K12, O12, O15, and O18. A: Silver stain; B-D, respectively: Immunoblots obtained with SZ 43 3.4.8 (R1-specific), H4 361.23 (R2 specific), W4 434.07 (R3 specific), and SZ $27 \quad 150.3$ (cross-reactive).
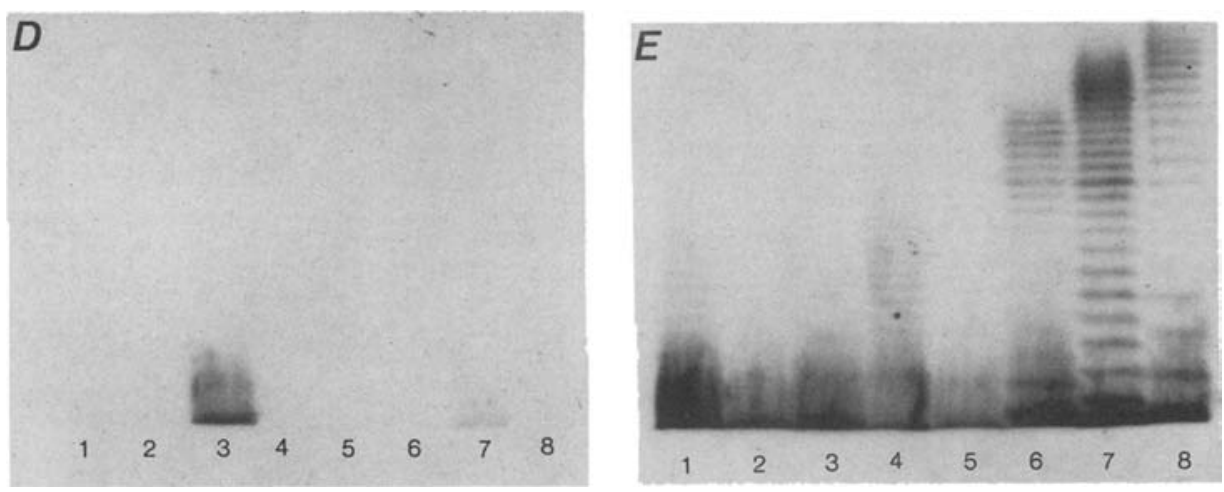

With the rough mutants, a band was seen that corresponded to the single band seen in the silver stain. With the smooth strains, the $\mathrm{R} 1$ and $\mathrm{R} 3$ MAbs again reacted with a single band corresponding to the fastest-moving band seen in the silver stain. This band consists of LPS core that has not been substituted with $\mathrm{O}$ antigen [13]. The $\mathrm{R} I$ antibody reacted in the same way in blots with the O1, O2, O4, O6, and 075 strains (not shown). The R2-specific MAb H4 361.23 and the cross-reactive MAb SZ 27150.3 reacted with the unsubstituted core band and also with the "ladder" pattern of bands corresponding to O-substituted LPS core. MAb SZ 27 150.3 reacted weakly in the blot with bands in the $\mathrm{R} I$ and R4 LPS preparations that were not visible in the silver stain. We believe these bands represent production, due to a leaky mutation, of small quantities of O-substituted LPS that are not detected by the silver stain but are detected by immunoblot.

Binding of anti-LPS core MAbs to gram-negative bacilli other than E. coli. Four Shigella strains were tested in ELISA, and all reacted with the cross-reactive MAb. In addition, both $S$. sonnei strains reacted with the R 1 -specific MAb, while the $S$. flexneri strains reacted with the R3-specific MAb. This is in agreement with previous studies of LPS core in these species [14-16]. Blood culture isolates of other gram-negative genera (Acinetobacter, 7; Citrobacter, 5; Enterobacter, 18; Klebsiella, 14; Proteus, 9; Providencia, 1; Pseudomonas, 8; Salmonella, 2; and Serratia, 5) were also tested in ELISA with the core type-specific and cross-reactive MAbs. The only positive reaction observed was between the two Salmonella isolates and the cross-reactive MAb. The cross-reactive MAb SZ 27150.3 was tested in an immunoblot against LPS (proteinase $\mathrm{K}$ extracts) from single blood culture isolates of $K$. pneumoniae, Proteus mirabilis, P. aeruginosa, Enterobacter cloacae, and Citrobacter freundii. No reaction was observed with any of these isolates, confirming the lack of reactivity seen in ELISA.

Frequency of MAb-defined core types in wild-type isolates of E. coli. E. coli isolates from blood cultures $(n=79)$, urine cultures ( $n=80)$, and fecal specimens from asymptomatic volunteers $(n=21)$ were tested in ELISA with core typespecific and cross-reactive MAbs. The cross-reactive MAb reacted with all of the isolates tested except for two of the urine isolates. When the strains reacting with the cross-reactive MAb were tested with the R1-, R2-, and R3-specific MAbs, each MAb reacted with a mutually exclusive group of isolates, which were classified as R1, R2, and R3, respectively. Figure 2 illustrates these results for the fecal isolates. Of the two urine isolates that did not react with the crossreactive antibody, one reacted with the $\mathrm{R} l$ antibody. These and the isolates that did not react with any of the core-specific antibodies are referred to as RNC (not classified). The numbers of wild-type isolates in the four categories are given in table 2. Rl was the most common classification overall $(123 / 181,68 \%)$ and was more common among isolates from 

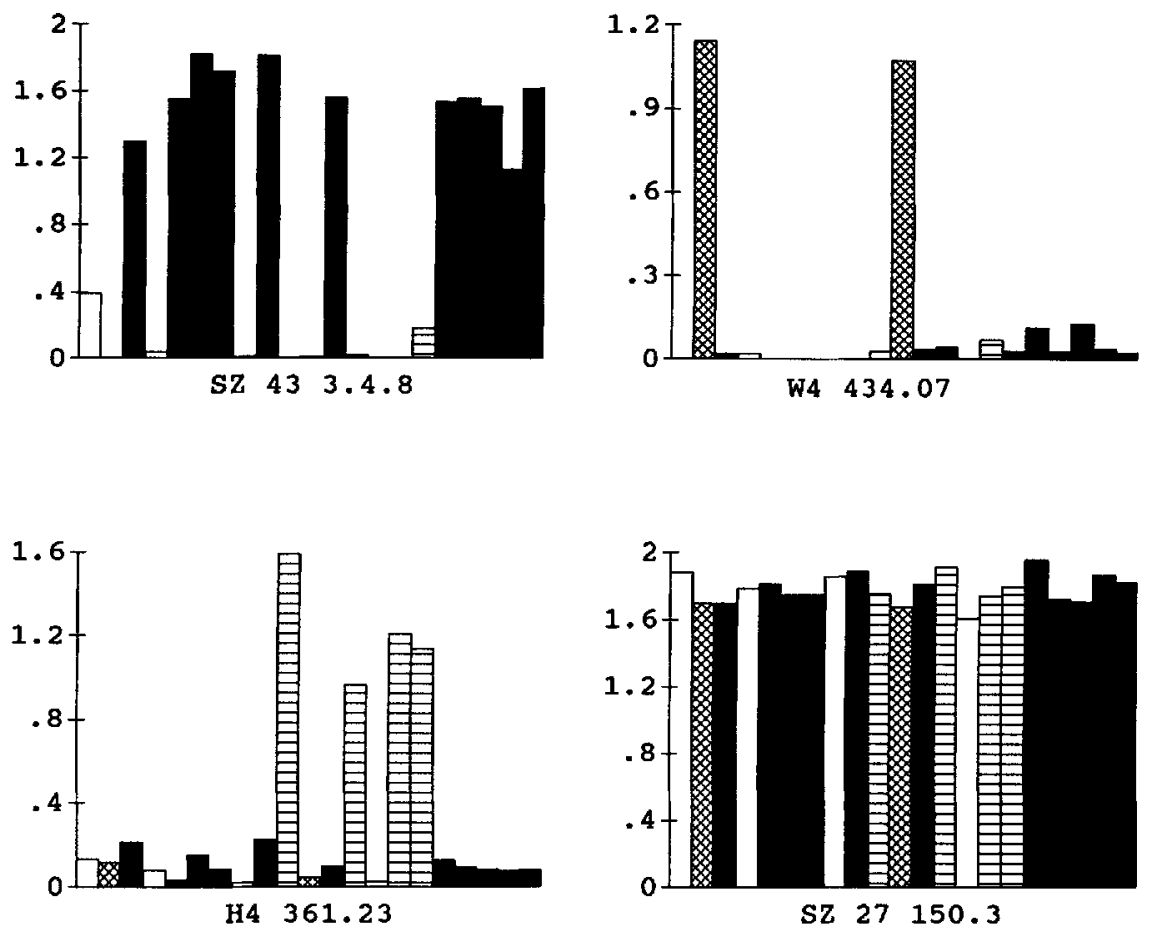

Figure 2. Reactivity (ELISA optical density) of antibodies with 21 fecal $E$. coli showing mutually exclusive reactions. Shading indicates core type to which each strain was assigned.

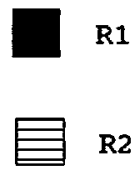

R3

R Not Classified

urine $(62 / 80,78 \%)$ than among those from blood culture $(48 / 79,61 \%, P=.017)$ or feces $(11 / 21,52 \%, P=.030)$.

Those MAbs that had reacted with R2, K12, and Salmonella rough mutants reacted with all of the R2 group of wildtype isolates but also reacted with variable numbers of the RNC isolates. This showed that the RNC group was heterogeneous but did not permit clear subdivision of this group.

Relationship between MAb-defined core type and sensitivity

Table 2. Frequency of core types in wild-type isolates of $E$. coli determined by binding of core-type-specific monoclonal antibodies (MAbs) in ELISA.

\begin{tabular}{|c|c|c|c|c|c|}
\hline \multirow[b]{2}{*}{ Source } & \multicolumn{4}{|c|}{$\begin{array}{l}\text { No. }(\%) \text { of strains assigned to MAb-defined } \\
\text { core type }\end{array}$} & \multirow[b]{2}{*}{ Total } \\
\hline & R 1 & $\mathrm{R} 2$ & R3 & $\mathrm{RNC}$ & \\
\hline Blood culture & $48(61)$ & $6(7)$ & $12(15)$ & $13(16)$ & 79 \\
\hline Urine & $64(81)$ & $4(5)$ & $4(5)$ & $8(10)$ & 80 \\
\hline Feces & $11(52)$ & $4(19)$ & $2(10)$ & $4(19)$ & 21 \\
\hline Total & $123(68)$ & $14(8)$ & $18(10)$ & $25(14)$ & 180 \\
\hline
\end{tabular}

NOTE. NC, not classified. to rough-specific and $K$-specific phages (table 3 ). The collection of wild-type $E$. coli was tested for sensitivity to the rough-specific phages that have been used to distinguish between the different $\mathrm{R}$ types of $E$. coli [3], since it was thought that the pattern of reactivity might support the MAb-defined $R$ typing scheme. Fifty of the 180 isolates tested were sensitive to one or more of the rough-specific phages. They were found among each of the R types defined with MAbs. They included isolates that were $\mathrm{O}$ typeable and produced a ladder

Table 3. Sensitivity of $180 \mathrm{E}$. coli of different core types to roughspecific, K1-specific, and K5-specific phages.

\begin{tabular}{|c|c|c|c|c|c|c|c|c|}
\hline \multirow{2}{*}{$\begin{array}{l}\text { Core type } \\
(n)\end{array}$} & \multicolumn{8}{|c|}{ No. of strains sensitive to phage } \\
\hline & Fo & $\operatorname{Br} 10$ & $\mathrm{C} 21$ & 6SR & T4 & Any $\mathrm{R}+$ & K1 & K5 \\
\hline $\mathrm{R} I(123)$ & 0 & 8 & 17 & 5 & 28 & 40 & 19 & 20 \\
\hline $\mathrm{R} 2$ (14) & 0 & 2 & 0 & 1 & 3 & 3 & 0 & 1 \\
\hline R3 (18) & 0 & 0 & 0 & 0 & 2 & 2 & 0 & i \\
\hline RNC (25) & 0 & 3 & 2 & 2 & 3 & 5 & 3 & 2 \\
\hline Total $(180)$ & 0 & 13 & 19 & 8 & 36 & 50 & 22 & 24 \\
\hline
\end{tabular}

NOTE. Any R+, any rough-specific phage; NC, not classified. 
Table 4. O type of $E$. coli of different core types from blood and urine cultures.

\begin{tabular}{lrllllllllllll}
\hline & \multicolumn{1}{c}{ No. of each core type associated with O type } \\
\cline { 2 - 11 } $\begin{array}{l}\text { Core type } \\
n\end{array}$ & O1 & O2 & O4 & O5 & O6 & 07 & 08 & 015 & 018 & O75 & NT \\
\hline R1 (112) & 11 & 0 & 6 & 1 & 26 & 3 & 3 & 0 & 10 & 6 & 46 \\
R2 (10) & 0 & 0 & 0 & 0 & 0 & 0 & 0 & 0 & 0 & 3 & 7 \\
R3 (16) & 0 & 0 & 0 & 0 & 0 & 0 & 0 & 3 & 0 & 0 & 13 \\
RNC (21) & 1 & 2 & 0 & 0 & 0 & 2 & 0 & 0 & 0 & 0 & 16 \\
$\quad$ Total (159) & 12 & 2 & 6 & 1 & 26 & 5 & 3 & 3 & 10 & 9 & 82 \\
\hline
\end{tabular}

NOTE. NT, not typed by available $\mathrm{O}$-specific sera; $\mathrm{NC}$, not classified.

pattern on silver stain of LPS, as was found by Cross et al. [6]. $\mathrm{C} 21$ sensitivity, a marker for the $\mathrm{R} 1$ rough mutant, was found in 19 isolates (17 RI and 2 RNC). FO sensitivity is a marker for $\mathrm{R} 2$ rough mutants, but no isolate was $\mathrm{FO}$ sensitive. There was no other discernible relationship between $R$ type and sensitivity to particular rough-specific phages.

Forty-six of the 180 isolates were sensitive to one of the two K-specific phages. The RI isolates accounted for 19 of the $22 \mathrm{Kl}$ isolates and 20 of the $24 \mathrm{~K} 5$ isolates found.

Relationship between MAb-defined core type and $O$ type among the urine and blood culture isolates. The urine and blood culture isolates of $E$. coli were tested for $12 \mathrm{O}$ types found commonly among $E$. coli from blood culture $[6,12]$ or urinary tract infection [17] (table 4). The overall proportion of isolates that belonged to one of these $O$ types was much higher among those of $\mathrm{R} 1$ core type $(66 / 112,58 \%)$ than among the other core types $(11 / 47,23 \%, P=.0005)$. Some constant associations between $\mathrm{O}$ type and core type were apparent: all 2606 isolates were $\mathrm{R} 1$, and all 3015 isolates were R3. In contrast, $\mathrm{O} 75$ isolates were found among both the $R 1$ and the $R 2$ core types. The proportion of the $R 1$ isolates that belonged to one of the common $O$ types was greater among the blood culture isolates $(32 / 48,67 \%)$ than among the urine culture isolates $(34 / 64,53 \%)$. This difference is not statistically significant $(P=.177)$, but the number of $\mathrm{R} 1$ isolates not belonging to the common $\mathrm{O}$ types could account for the greater proportion of $\mathrm{R} l$ isolates among the isolates from urine.

Relationship between $R$ type, serum resistance, and LPS chemotype among blood culture isolates. Serum resistance, which is a property of most invasive strains of $E$. coli [6], was determined for all the blood culture isolates; 21 (26\%) of 79 were serum sensitive. There were serum-sensitive isolates of all the core types, but serum sensitivity was slightly less common among the $\mathrm{R} 1$ isolates $(9 / 48,19 \%)$ than among the other $\mathrm{R}$ types (10/31, 32\%). This difference may have been due to the association of $\mathrm{R} 1$ core with $\mathrm{K} 1$ and $\mathrm{K} 5$ capsule, since all of the $11 \mathrm{~K} 1$ isolates and all but 1 of the $10 \mathrm{~K} 5$ isolates from blood cultures were serum resistant.

Seventy of the 79 blood culture isolates produced a typical ladder pattern of LPS in silver stains of proteinase $\mathrm{K}$ digests (data not shown). The other 9 showed only one or a few fast-moving bands, one corresponding to the unsubstituted core. Three isolates were R1, 3 were R2, 2 were $R 3$, and 1 was $\mathrm{RNC}$.

\section{Discussion}

We have described a group of MAbs that are selective for the LPS core of E. coli. Some of the MAbs also bind to Salmonella and Shigella strains that share E. coli core structures. The MAbs do not bind to a range of other gram-negative bacilli, confirming that the binding observed was specific. We have shown that the binding of these antibodies in ELISA to bacterial cells heated at $100^{\circ} \mathrm{C}$ corresponds to their binding to unsubstituted (and in some cases O-substituted) LPS core in immunoblots.

Unsubstituted LPS core is present in smooth bacterial cells but may not be readily accessible to MAbs in standard laboratory cultures $[18,19]$. Core epitopes are, however, much more accessible on bacterial cells grown in serum or in a magnesium-depleted medium, which is thought to mimic the conditions in the infected host [20]. Core epitopes also become more accessible in bacterial preparations heated at $100^{\circ} \mathrm{C}[21,22]$. The expression and accessibility of core epitopes and the possible existence of cross-reactive epitopes have important implications for immunotherapy of gramnegative sepsis [23].

We have examined the binding of selected MAbs to heated bacterial cells in ELISA. We could recognize three mutually exclusive groups among $E$. coli isolates and labeled them R1, R2, and R3 after the corresponding rough mutants. A fourth group, which could not be assigned to a particular core type, was referred to as RNC and is probably of heterogeneous core type. We included in the $\mathrm{RNC}$ group the strain that reacted with the $\mathrm{R} 1$-specific antibody but not with the cross-reactive MAb. If this strain had been of $R I$ core type, a reaction with the cross-reactive MAb would have been expected.

We anticipate that each of the groups R1, R2, and R3 defined with MAbs probably has the core structure of the named rough strain, though this has not been confirmed by chemical analysis. The presence of $\mathrm{C} 21$ sensitivity (a marker for $\mathrm{R} I$ rough mutants) among the wild-type isolates of MAbdefined $R 1$ rather than $R 2$ or $R 3$ core type supports our view that the MAb-defined core types are the same as the reference rough strains. Two RNC isolates were also $\mathrm{C} 21$ sensitive, but the specificity of these phages for particular core types is not based on an independent knowledge of their receptor sites, and it is not known how they might react with as-yet-unidentified core types of $E$. coli. Phage typing is of little value in determining the core type of wild-type isolates since most are resistant to all of the phages used.

No isolates of R4 or K12 core type were positively identi- 
fied. This may be explained by the lack of suitable specific MAbs. We did have MAbs reactive with $\mathrm{K} 12$, but these also reacted with $R 2$ and did not bind to a clearly defined group of wild-type isolates other than those that reacted with the R2-specific MAbs. The RNC group may include isolates with $\mathrm{R} 4$ or $\mathrm{K} 12$ core but probably also contains one or more as-yet-unidentified core types.

Comparison of the structural formulas [15] with the specificities of our MAbs could suggest binding of the MAbs to particular sugar residues. RI and R3 each have a unique terminal branching hexosyl unit ( $\beta 1-3-$ linked glucose and $\alpha$ I-3-linked $N$-acetylglucosamine, respectively) that may be the epitope for the R1 and R3 antibodies. R2 and Salmonella core share a terminal $\alpha 1$-2-linked $N$-acetylglucosamine, while R2 and K12 have in common the next three sugar residues. These may therefore be the epitopes for the MAbs that bound to these combinations of strains. A unique binding site for the R2-specific antibody is not obvious from the structural formulas. From the calculated three-dimensional structure of the different core types [24], however, it appears that the branch-terminating $\alpha 1-6$ galactose present in R2, Salmonella core, and K12 may be in a different orientation in R2 and may therefore be the R2-specific epitope. It is of note that while most of the core-specific MAbs reacted in immunoblot only with unsubstituted core, the R2-specific $\mathrm{MAb}$ also reacted with $\mathrm{O}$-substituted core, giving a ladder pattern in immunoblots. This implies that the R2-specific epitope is the only one not altered or masked by the attachment of the $O$ polysaccharide. Further study of the binding site of these antibodies may therefore be useful in elucidating the three-dimensional structure of LPS core and in attempts to define the attachment site of the $O$ polysaccharide.

The binding of the cross-reactive MAb SZ 27150.3 to almost all of the $E$. coli isolates and to the Salmonella and Shigella strains, together with the pattern of binding of the core-specific MAbs, supports the view that these organisms share closely related LPS cores. The lack of binding of any of the antibodies to other genera suggests that these core structures are not distributed more widely. The structure of LPS core has not been determined for all the other genera, but it has been shown that the LPS cores of Citrobacter [25, 26] Proteus [27], and Pseudomonas [28] species are different from that of $E$. coli.

A MAb designated T6, which recognized the R2 core of $E$. coli and the core of Salmonella species, did not react with 25 clinical isolates of $E$. coli in Hong Kong [29], suggesting that the $\mathbf{R} 2$ core type was rare. We have found, however, that $\mathbf{R} 2$ accounted for $8 \%$ of our collection of $E$. coli isolates. This suggests that attempts to use MAb T6 in a capture ELISA [22] to detect Salmonella organisms in clinical samples may yield a significant number of false-positives because of the presence of $E$. coli of R2 core type.

The tendency for certain apparently distinct properties of E. coli to occur together [5] seems to apply to the relationship between core types and $\mathrm{O}$ and $\mathrm{K}$ types. It may be that there are chemical or structural factors limiting the ability of $E$. coli to express certain combinations of $\mathrm{O}$ and core types (e.g., $\mathrm{O} 15$ and R I) or it may be that some such combinations have a selective advantage in the host. The finding that there was greater proportion of $\mathrm{R} I$ core type among the urine isolates and in particular that there was a greater proportion of $\mathrm{R} I$ isolates not belonging to one of the common $O$ types may indicate some relationship between $\mathrm{Rl}$ core type and unusual $O$ types and pathogenicity in the urinary tract. Further studies are needed before firm conclusions can be drawn on this point.

Core typing with MAbs may be a useful addition to current typing methods for $E$. coli. The number of possible types is small, and the core type does not usually discriminate among strains of a given $\mathrm{O}$ and $\mathrm{K}$ type, but core typing may be useful in recognizing relatedness between groups of $E$. coli. We hope to use these antibodies to investigate the LPS core type of a wider range of Shigella and E. coli isolates from a variety of sources.

\section{Acknowledgments}

We thank Loraine McMillan, Keith James, and colleagues in the Department of Surgery, Edinburgh University, and at Sandoz Pharma for performing cell fusions and producing selected antibodies; Boyd Scott, Scottish National Blood Transfusion Service, for screening cell fusions for LPS antibodies; and Frances McLoughlin and Linda Milne for assistance in growing bacteria and ELISA.

\section{References}

1. Schmidt G, Jann K, Jann B. Immunochemistry of R lipopolysaccharides of Escherichia coli. Different core regions in the lipopolysaccharides of $O$ group 8. Eur J Biochem 1969;10:501-10.

2. Schmidt G, Fromme I, Mayer H. Immunochemical studies on core lipopolysaccharides of Enterobacteriaceae of different genera. Eur $\mathbf{J}$ Biochem 1970; 14:357-66.

3. Schmidt G, Jann B, Jann K. Genetic and immunochemical studies on Escherichia coli O14:K:H-. Eur J Biochem 1974;42:303-9.

4. Jansson PE, Lindberg B, Lindberg AA, Wollin R. Structural studies on the hexose region of Enterobacteriaceae type- $R 3$ core polysaccharide. Carbohydr Res 1979;68:385-9.

5. Ørskov F, Whittam TS, Cravioto A, Ørskov I. Clonal relationships among classic enteropathogenic Escherichia coli (ETEC) belonging to different groups. J Infect Dis 1990;162:76-81.

6. Cross AS, Gemski P. Sadof JC, Ørskov F, Ørskov I. The importance of $\mathrm{KI}$ capsule in invasive infections caused by Escherichia coli. $\mathrm{J}$ Infect Dis 1984; 149: 184-93.

7. Kipps TJ, Hertzenberg LA. Schemata for production of monoclonal antibody-producing hybridomas. In: Weir DM, ed. Handbook of experimental immunology. 4th ed. Oxford: Blackwell, 1986:108.1-.9.

8. Scott BB, Barclay GR, Smith DGE, McLoughlin F. Poxton IR. IgG antibodies to gram-negative endotoxin in human sera. 1. Lipolysaccharide (LPS) cross-reactivity due to antibodies to LPS core. Serodiagn Immunother Infect Dis 1990;4:25-38.

9. Hitchcock PJ, Brown TM. Morphological heterogeneity among Salmo- 
nella lipopolysaccharide chemotypes in silver-stained polyacrylamide gels. J Bacteriol 1983;154:269-77.

10. Laemmli UK. Cleavage of structural proteins during the assembly of the head of bacteriophage T4. Nature 1970:277:680-5.

11. Towbin H, Staehelin T, Gordon J. Electrophoretic transfer of proteins from polyacrylamide gels to nitrocellulose sheets: procedure and some applications. Proc Natl Acad Sci USA 1979;76:4350-4.

12. Ørskov F. Ørskov I. Escherichia coli O:H serotypes isolated from human blood. Acta Pathol Microbiol Immunol Scand 1975;83:595-600.

13. Palva ET, Mäkela PH. Lipopolysaccharide heterogeneity in Salmonella typhimurium analyzed by sodium dodecyl sulphate/polyacrylamide gel electrophoresis. Eur J Biochem 1980;107:137-43.

14. Mayer $H$, Schmidt $G$. The occurrence of three different lipopolysaccharide cores in Shigella and their relationship to known enterobacteria! core types. Zentralbl Bakteriol Mikrobiol Hyg [A] 1973;224:34554.

15. Jansson PE, Lindberg AA, Lindberg B, Wollin R. Structural studies on the hexose region of the core in lipopolysaccharides from Enterobacteriaceae. Eur J Biochem 1981;1 15:571-7.

16. Gamian A, Romanowska E. The core structure of Shigella sonnei lipopolysaccharide and the linkage between $O$ specific polysaccharide and the outer core region. Eur J Biochem 1982;129:105-9.

17. Grüneberg RN, Leigh DA. Brumfitt W. Escherichia coli serotypes in urinary tract infection: studies in domiciliary, antenatal and hospital practice. In: O'Grady FO, Brumfiit W, eds. Urinary tract infection. London: Oxford University Press, 1968:68-79.

18. Nelson D, Neill W, Poxton IR. A comparison of immunoblotting, flow cytometry and ELISA to monitor the binding of anti-lipopolysaccharide monoclonal antibodies. J Immunol Methods 1990;133:227-33.

19. Gigliotti F, Shenep JL. Failure of monoclonal antibodies to core glycolipid to bind intact smooth strains of Escherichia coli. J Infect Dis 1985;151:1005-11.

20. Nelson D, Bathgate AD, Poxton IR. Monoclonal antibodies as probes for detecting lipopolysaccharide expression on Escherichia coli from different growth conditions. J Gen Microbiol 1991;137:2741-51.

21. Nelson D. The expression and detection of Escherichia coli lipopolysaccharide with monoclonal antibody probes [PhD thesis]. Edinburgh. UK: Edinburgh University, 1991:241-8.

22. Choi D, Tsang RSW, Ng MH. Sandwich capture ELISA by a murine monoclonal antibody against a genus-specific LPS epitope for the detection of different common serotypes of salmonellas. J Appl Bacteriol 1992;72:134-8.

23. Mitov IG, Terziiski DG. Immunoprophylaxis and immunotherapy of gram-negative sepsis and shock with antibodies to core glycolipids and lipid A of bacterial lipopolysaccharides. Infection 1991;19:38390.

24. Jansson PE, Wollin R. Bruse GW, Lindberg AA. The conformation of core oligosaccharides from Escherichia coli and Salmonella typhimurium lipopolysaccharides as predicted by semi-empirical calculations. J Mol Recognit 1989;2:25-36.

25. Romanowska E, Gamian A, Dabrowski J. Core region of Citrobacter lipopolysaccharide from strain PCM1487. Eur J Biochem 1986; 161:557-64

26. Romanowska E, Gamian A, Lugowski C, et al. Structural elucidation of the core regions from Citrobacter $\mathrm{O} 4$ and $\mathrm{O} 36$ lipopolysaccharide by chemical and enzymatic methods, gas chromatography/mass spectrometry and NMR spectroscopy at $500 \mathrm{MHz}$. Biochemistry 1988;27:4153-61.

27. Radziejewska-Lebrecht J, Mayer H. The core region of Proteus mirabilis R1 10/1959 lipopolysaccharide. Eur J Biochem 1989:183:57381.

28. Rowe PSN, Meadow PN. Structure of the core oligosaccharide from the lipopolysaccharide of Pseudomonas aeruginosa PACIR and its defective mutants. Eur J Biochem 1983;132:329-57.

29. Tsang RSW, Chan KH, Chau PY, Wan KC, Ng MH, Schlecht S. A murine monoclonal antibody specific for the outer core oligosaccharide of Salmonella lipolysaccharide. Infect Immun 1987;55:21 1-6. 